\title{
Immunoglobulin Treatment in Severe Ovarian Hyperstimulation Syndrome
}

\author{
Gemma Grau Gómez ${ }^{1}$, Oriol Giménez Campanario ${ }^{2}$, Nuria Pons Serra², Mireia Cairó Llobell ${ }^{1}$, Pedro Almagro Mena ${ }^{1}$ \\ ${ }^{1}$ Department of Internal Medicine, Fundació Mútua de Terrassa, Terrassa, Spain \\ ${ }^{2}$ Department of Obstetrics and Gynecology, Fundació Mútua de Terrassa, Terrassa, Spain
}

Doi: 10.12890/2019_001253 - European Journal of Case Reports in Internal Medicine - @ EFIM 2019

Received: $13 / 08 / 2019$

Accepted: $22 / 08 / 2019$

Published: 02/10/2019

How to cite this article: Grau Gómez G, Giménez Campanario O, Pons Serra N, Cairó Llobell M, Almagro Mena P. Immunoglobulin treatment in severe ovarian hyperstimulation syndromeEJCRIM 2019;6: doi:10.12890/2019_001253.

Conflicts of Interests: The Authors declare that there are no competing interest

This article is licensed under a Commons Attribution Non-Commercial 4.0 License

\section{ABSTRACT}

A 32-year-old woman undergoing an in vitro fertilization program was admitted to our hospital with the diagnosis of severe ovarian hyperstimulation syndrome (OHSS). Transvaginal ultrasonography showed two gestational sacs. Treatment with fluid restriction, serum albumin and intravenous furosemide was started, and repeated thoracentesis and paracenteses were performed. In the absence of clinical improvement, the patient was transferred to the intensive care unit and a therapeutic abortion was suggested. Due to the similarities between OHSS and idiopathic systemic capillary leak syndrome, we offered the patient compassionate treatment with intravenous immunoglobulins. After administration, the patient showed rapid improvement and we were able to suspend intravenous furosemide and serum albumin. She was discharged, and pregnancy has continued normally to date.

\section{LEARNING POINTS}

- OHSS is an infrequent yet serious complication of assisted reproductive treatment that involves increased capillary permeability.

- In severe refractory cases, immunoglobulins may be a useful and safe treatment to reverse this syndrome.

\section{KEYWORDS}

Severe ovarian hyperstimulation syndrome, immunoglobulins

\section{CASE DESCRIPTION}

A 32-year-old woman with hereditary optic atrophy due to mutation of the OPA1 gene was admitted to hospital for dyspnoea. The patient was in an in vitro fertilization program. She had been treated with FSHr and GnRH for ovarian stimulation with subsequent administration of HCGr and implantation of two embryos free of the OPA1 mutation, 5 days before admission. Physical examination revealed grade 2 ascites and bilateral lower limb oedema. Blood analysis showed haemoglobin of $16.6 \mathrm{~g} / \mathrm{dl}$, sodium $133 \mathrm{mmol} / \mathrm{l}$, albumin $3.3 \mathrm{~g} / \mathrm{l}$ and $\beta \mathrm{HCG} 200 \mathrm{mU} /$ $\mathrm{ml}$, with preserved renal and hepatic function. Transvaginal ultrasonography demonstrated enlarged ovaries with free fluid in the pelvis and two gestational sacs. Following the diagnosis of OHSS, fluid restriction and thromboembolic prophylaxis were started. On the sixth day after admission, abdominal distension and dyspnoea increased, together with hypotension and signs of haemoconcentration. Ultrasonography demonstrated severe pleural effusion and ascites. Electrocardiogram and echocardiogram findings were within the normal range. Serum albumin and intravenous furosemide were added, alongside repeated paracentesis and thoracentesis. Given the persistence of dyspnoea with refractory pleural effusion, a thoracic drainage tube was placed, and the patient was transferred to the intensive care unit. In the following days symptoms persisted and a therapeutic abortion was suggested. Given the similarities between OHSS and idiopathic systemic capillary leak syndrome (SCLS), we offered the patient compassionate treatment with intravenous immunoglobulins (IVIG) 
which has previously proven useful in SCLS. After administration of IVIG ( $0.5 \mathrm{~g} / \mathrm{kg} /$ day for 3 consecutive days), the patient showed rapid improvement, and we were able to suspend intravenous furosemide and serum albumin and to remove the thoracic drainage tube. The patient was discharged, and pregnancy has continued normally to date.

\section{DISCUSSION}

OHSS is the most severe complication associated with assisted reproductive treatments. Its pathophysiology involves increased capillary permeability that leads to a wide variety of signs and symptoms such as hypotension, ascites and pleural effusion. Although it is generally self-limiting, $5 \%$ of patients will develop life-threatening complications ${ }^{[1]}$. There is no specific treatment, and management is based on the control of fluid balance, thromboembolic prophylaxis, and paracentesis or thoracentesis when needed.

As a result of this increase in capillary permeability to fluids and proteins, there is a loss of protein to fluid in the interstitial space with depletion of intravascular volume, associated with overproduction of vasoactive cytokines and vascular endothelial growth factor (VEGF). This is common to other human diseases that can also cause capillary leak syndrome such as sepsis, several drugs and SCLS ${ }^{[2]}$.

We used IVIG because of the similarities with SCLS, a rare entity characterized by recurrent episodes of capillary leak syndrome with high mortality secondary to hypovolemic shock, acute renal failure and life-threating pulmonary oedema during the recovery phase. SCLS is usually associated with a monoclonal gammopathy of uncertain significance. As in OHSS, cytokines and VEGF were increased in serum from acutely ill subjects with SCLS ${ }^{[3]}$. Several reports and cohort studies have shown the efficacy of IVIG both in the acute phase and in the prevention of recurrence ${ }^{[4,5]}$. Although the exact mechanism of action is unknown, IVIG have multiple activities including inhibitory effects on cytokine and VEGF production, and have been used to treat autoimmune, neurological and haematological diseases. IVIG are generally well tolerated and their administration during pregnancy is safe. Although new studies are needed to confirm their usefulness, the present case suggests that IVIG may be a useful and safe treatment for severe cases of OHSS refractory to conventional management.

\section{REFERENCES}

1. Selter J, Wen T, Palmerola KL, Friedman AM, Williams Z, Forman EJ. Life-threatening complications among women with severe ovarian hyperstimulation syndrome. Am J Obstet Gynecol 2019;220(6):575.e1-575.e11.

2. Siddall E, Khatri M, Radhakrishnan J. Capillary leak syndrome: etiologies, pathophysiology, and management. Kidney Int 2017;92:37-46.

3. Xie Z, Ghosh CC, Parikh SM, Druey KM. Mechanistic classification of the systemic capillary leak syndrome: Clarkson disease. Am J Respir Crit Care Med 2014;189(9):1145-1147.

4. Pineton de Chambrun M, Gousseff M, Mauhin W, et al. Intravenous immunoglobulins improve survival in monoclonal gammopathy-associated systemic capillary-leak syndrome. Am J Med 2017;130(10):1219.e19-1219.e27.

5. Almagro P, Martí JM, Garcia Pascual L, Rodriguez-Carballeira M. Successful treatment of systemic capillary leak syndrome with intravenous immunoglobulins. Rev Clin Esp 2012;212(4):218-219. 